\title{
Factors Influencing Adherence to Preventive Behaviors among Thais with Hypertension: A Literature Review
}

\author{
Siriwan CHUKUMNIRD ${ }^{1, *}$, Umaporn BOONYASOPUN ${ }^{2}$ \\ and Krittaporn SIRISOM ${ }^{1}$
}

\author{
${ }^{1}$ Boromarajonani College of Nursing, Songkhla, Songkhla 90000, Thailand \\ ${ }^{2}$ Prince of Songkla University, Hat Yai, Songkhla 90112, Thailand
}

('Corresponding author's e-mail: scsiriwanch@gmail.com)

Received: 1 May 2017, Revised: 8 May 2018, Accepted: 4 June 2018

\begin{abstract}
The purpose of this study was to summarize empirical evidence regarding factors influencing adherence to preventive behaviors of Thais with hypertension. An electronic search was performed using PubMed, Medline, CINAHL, SCOPUS, ThaiJo, and ThaiLis databases to identify studies that examined the factors which influenced adherence to preventive behaviors in Thai adults with hypertension. We identified research studies published between 1995 and 2017, which were assessed by 2 independent reviewers. Fourteen publications were included; 3 of these were qualitative studies. Social support, knowledge of hypertension, perception of illness, perceived benefits, perceived barrier, perceived selfefficacy, Thai cultural values, and patient-provider communication were identified as factors influencing adherence to preventive behaviors of Thai adults with hypertension. Although information about factors influencing adherence is growing, more research is needed on how to provide the best intervention support for this challenging task.
\end{abstract}

Keywords: Adherence, preventive behaviors, hypertension, Thai patients, review

\section{Introduction}

Hypertension is a major worldwide public health concern because of its high prevalence and concomitant risks for stroke, heart disease, and kidney disease [1,2]. However, more than half the people with hypertension worldwide were not aware of their elevated blood pressure [1]. The prevalence of hypertension is increasing in every country, particularly in low and middle-income countries [3]. About 1.13 billion people across the world are living with high blood pressure [3]. In Thailand, the previous national health and nutrition examination surveys (NHANES IV and V) found that the prevalence of hypertension in adults 18 years and older in 2008 was $21.4 \%$ but by 2014 it had risen to $24.47 \%$ [4,5]. These surveys also reveal improvements in awareness, treatment and control of hypertension in the past decade. However, the level of awareness, treatment and high blood pressure control is still unsatisfactory. Almost half of Thais with hypertension were not aware of their blood pressure condition. Only one-fifth of Thais within the country succeed in maintaining their blood pressure to within normal limits [5]. Promoting a high level of adherence to treatment regimens of Thai people is essential for treating hypertension.

Hypertension requires long-term treatment which usually involves pharmacological and lifestyle modification therapies. The guideline updates of The Seventh Report of the Joint National Committee on Prevention, Detection, Evaluation, and Treatment of High Blood Pressure (JNC 7) [6] identify lifestyle modifications as critical to hypertension control. Recommended lifestyle modification for people with hypertension include increasing physical activity, achieving or maintaining appropriate body weight, 
http://wjst.wu.ac.th

moderation in alcohol intake, and adoption of dietary lifestyle modifications such as restriction of sodium intake and following the Dietary Approaches to Stop Hypertension (DASH) diet combined with stress management [6]. Several clinical trials have been confirmed on the usefulness of preventive behaviors in improving hypertension control, reducing cardiovascular risk and enhancing antihypertensive medication efficacy [7,8]. Hypertensive patients are advised to participate in preventive behaviors in order to lessen the disease burden and reduce the morbidity and mortality associated with cardiovascular complications [6]. Despite many efforts to promote antihypertensive measures, hypertension is also marked by uncontrolled high blood pressure and complications, and the leading cause for these problem is nonadherence to preventive behaviors.

The extent of preventive behavior recommendations can be explained by the concept of adherence. Adherence to long-term therapy is described by the WHO [9] as "the extent to which a person's behavior in terms of taking medications, following diets or executing lifestyle changes corresponds with agreed recommendations from the health care provider. Therefore, adherence to preventive behaviors in this review is defined as a behavior to follow preventive behaviors as prescribed by health care providers. Non-adherence to preventive behaviors implies the extent to which patients did not follow preventive behavior recommendations from health care providers.

Non-adherence to preventive behaviors in people with hypertension leads to increased premature progression of non-communicative diseases which increases health care costs and mortality [9]. Despite the different operational definitions of adherence to preventive behaviors used by researchers, the rates of non-adherence to preventive behavior recommendations among Thais with hypertension were unsatisfactory. A previous national survey (NHANES IV) $(n=5,733,872)$ reported that $22.9 \%$ people with hypertension did not control their daily salt intake, $26.3 \%$ did not control fat intake, $32.2 \%$ did not do any exercise, $37.6 \%$ did not control their weight, $14.1 \%$ smoked, and 19.1 consumed alcohol [4]. A previous cross-sectional survey $(\mathrm{n}=31,088)$ reported that $58.5 \%$ did not do any exercise, $45.85 \%$ smoked, $35.7 \%$ did not control daily fat intake, $44.1 \%$ consumed alcohol, and $62.3 \%$ did not control their weight [10]. Overall, the level of adherence to preventive behaviors of Thai people is poor and has confirmed previous reports on inadequate adherence to preventive behaviors among hypertensive patients in other parts of the world [11].

It is important to understand the factors influencing adherence to preventive behaviors among hypertensive patients. To date, there has been no review which summarizes the factors associated with adherence to preventive behaviors for Thai adults with hypertension. Since culture and beliefs can influence patients' responses in their lifestyle and illnesses behavior, the extent of adherence to preventive behaviors and the factors influencing them may be different in different populations. This review of the literatures will address this gap in the literature by analyzing empirical evidence regarding factors influencing adherence to preventive behaviors of Thais with hypertension.

\section{Methods}

A comprehensive literature review was conducted using the available resources including PubMed, Medline, SCOPUS, CINAHL, ThaiJo, and ThaiLis databases. A manual search was also conducted. The review inclusion criteria were dissertations and articles that (1) examined the factors of adherence to lifestyle modifications, (2) recruited Thai adults with hypertension, (3) conducted using quantitative and/or qualitative research designs, and (4) were written in English or Thai between the years 1995 and 2017. The keywords used in the research include "preventive behavior", "lifestyle modification", "exercise", "dietary control", "weight loss", "moderation of alcohol intake", "smoking cessation", "adherence", "non-adherence", "compliance", "non-compliance", "hypertension", "factor", "reason", "predictor", "determinant", "aspect", "component", and "element".

Subsequently, factors influencing adherence to preventive behaviors in this population of Thai patients were categorized according to the 5 dimensions of adherence model proposed by WHO [9]. This includes patient-related factors, social and economic factors, condition related factors, therapy-related factors, and health care team and system-related factors [9]. The WHO defines patient related factors as the perceptions, knowledge, beliefs, and attitudes of the patient; social and economic factors as social 
/economic factors; condition-related factors characterize particular illness related demands faced by the patient; therapy-related factors as complexity of the lifestyle modification regimens, duration of the lifestyle modifications, previous lifestyle modification failures, the immediacy of beneficial effects on lifestyle modifications; health care team and system-related factors as the effects of patient-provider relationship and communication on adherence.

45 articles identified through database searching

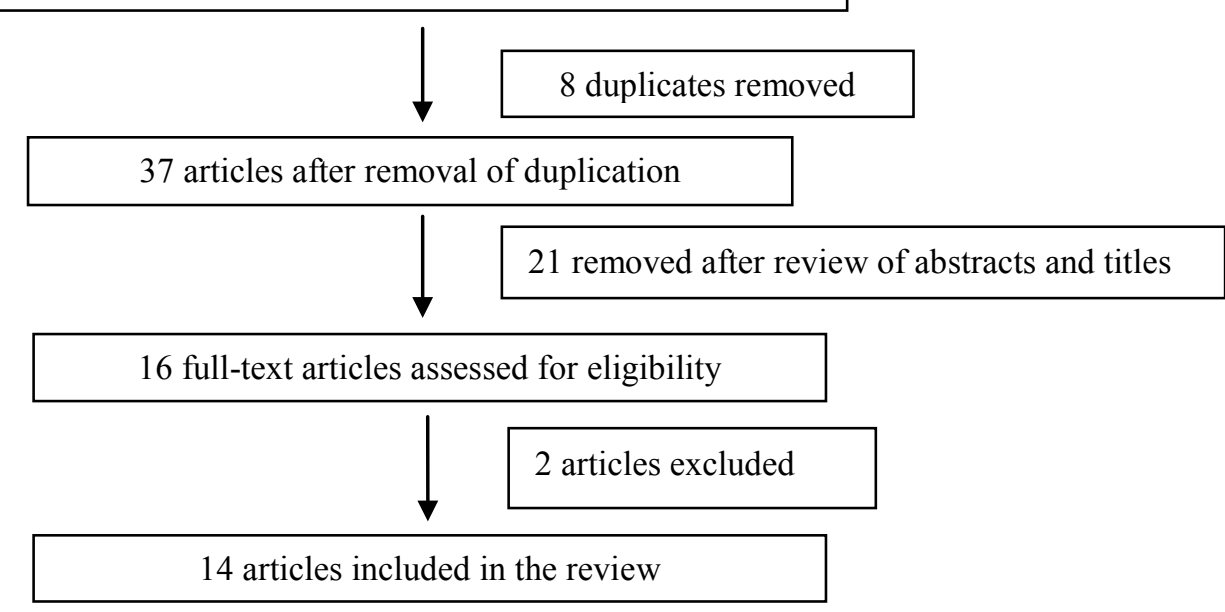

Figure 1 Summary of procedure in this study.

\section{Results}

A total of 45 studies were identified through database search. After screening the abstracts and reviewing the full text, the panel members determined that 14 research studies met the inclusion criteria. The procedure in this study is illustrated in the flowchart (Figure 1). These publications were then finally included and data were extracted and categorized based on the WHO's 5 dimensions of adherence as shown in Table 1. Each factor was examined for any established association with adherence to preventive behaviors. In particular, each factor required at least 2 articles to ensure a relationship with adherence to preventive behaviors. Among the 14 articles included, the majority were quantitative studies. Adherence to preventive behavior measures used in these studies were gathered from predominantly subjective data via self-reported questionnaires and interviews. Only 2 of 14 studies used self-report measures of food intake and smoking cessation behaviors. The methodological quality of each study was assessed by the critical appraisal checklist form developed by the Joanna Briggs Institute (JBI) [12].

Empirical evidence suggests that adherence to preventive behaviors for Thai adults with hypertension is influenced by numerous factors and these can be classified into 3 categories; social and economic factors, patient-related factors, and health team and system related factors [9].

\section{Social and economic factors}

Social and economic factors from the WHO adherence model are significant predictors of adherence to preventive behaviors. Six studies have explored hypertensive Thais' beliefs about social support and adherence to preventive behaviors $[14,15,17,18,19,26]$. Social support is consistently well documented as having favorable effects on preventive behavior practices. It is potentially important in helping patients positively initiate and practice preventive behaviors as evidenced in a qualitative literature [26]. A cross- 
http://wjst.wu.ac.th

sectional study found that social support had a positive relationship to adherence to preventive behaviors [19]. One quasi-experimental study revealed that community and family members were seen as motivators in adhering to preventive behaviors [15]. Moreover, an experimental study found that participating in the program using community participation had higher scores for stress management compared to the knowledge group [17]. However, 2 cross-sectional studies reported no significant findings $[14,18]$. Cross-sectional studies generally provide a lower level of evidence than experimental studies because they are usually unable to control for confounding variables. Studies examining social support generally assessed adherence to preventive behavior practices using self-report questionnaires.

\section{Patient-related factors}

Patient-related factors were found to be a predictor of adherence to preventive behaviors. Ten studies revealed that factors unique to an individual include, knowledge of hypertension, perception of illness, perceived self-efficacy, perceived benefits, perceived barriers, and Thai cultural values $[13,14,18,19,21-23,25-27]$.

\section{Knowledge of hypertension}

Four studies explored the association between knowledge about hypertension and adherence to preventive behaviors of Thai adults with hypertension. The results of these studies were inconsistent. Two cross-sectional studies revealed an association between knowledge of hypertension and adherence to preventive behaviors of hypertensive Thai adults [14,22]. One qualitative study also reported that knowledge of hypertension as well as healthy lifestyle plays an important role in the attitudes and beliefs of hypertensive Thai patients [26]. Conversely, a cross-sectional study reported no association between knowledge of hypertension and adherence to preventive behaviors of hypertensive Thai adults [19]. This study were conducted in only one province in the north of Thailand which lessened the external validity of the study and therefore, decreased the generalizability of the findings. In these quantitative studies, adherence to preventive behaviors was measured with questionnaires $(n=3)$.

\section{Perception of illness}

Four studies revealed that adult Thai patients' responses to adherence to preventive behavior treatment differ depending on their beliefs about their health condition [13,14,18,26]. One qualitative study indicated that illness perception is an important determinant for adherence to lifestyle modifications. Thais are not aware of their elevated blood pressure status because they perceive hypertension not as a disease [26]. In this qualitative study, the researcher did not measure adherence to preventive behaviors. Two cross-sectional studies supported qualitative findings and reported that perceptions about hypertension had a relationship to adherence regarding preventive behaviors of hypertensive Thai adults [14,18]. Patients' perceptions of hypertension have an important role in adherence to self-care behaviors such as following preventive behavior regimens [14]. Jianvitayakij et al. [18] found that concern about the harmful effects of smoking was a significant predictor of smoking cessation. Furthermore, an experimental study mentioned that patients who have clear and correct perceptions about hypertension are more likely to make appropriate decisions about adherence to sodium reduction [13]. Researchers assessed adherence to preventive behaviors using self-report of food intake during the previous $24 \mathrm{~h}(\mathrm{n}=1)$ and smoking cessation behavior $(\mathrm{n}=1)$. One cross-sectional study used self-report questionnaires. 
Table 1 Summary of studies examining factors influencing adherence to preventive behaviors of Thai adults with hypertension.

\begin{tabular}{|c|c|c|c|c|c|c|c|}
\hline $\mathbf{S} / \mathbf{N}$ & Author (year) & Study design & Sample & Independent variable & $\begin{array}{c}\text { Adherence to } \\
\text { preventive behaviors } \\
\text { measure }\end{array}$ & Data analysis & Results \\
\hline 1 & $\begin{array}{l}\text { Chonsin, } \\
\text { Kaveevivitchai, } \\
\text { Neelapaichit, \& } \\
\text { Piaseu, 2016. } \\
\text { [13] }\end{array}$ & $\begin{array}{l}\text { Two-group } \\
\text { experimental } \\
\text { research }\end{array}$ & $\begin{array}{l}64 \text { Thais with } \\
\text { essential } \\
\text { hypertension }\end{array}$ & $\begin{array}{l}\text { Perceived benefits, } \\
\text { Perceived barriers, } \\
\text { Perceived severity, } \\
\text { Perceived susceptibility }\end{array}$ & $\begin{array}{l}\text { Self-report: A form } \\
\text { for recording food } \\
\text { intake during the } \\
\text { previous } 24 \mathrm{~h}\end{array}$ & $\begin{array}{l}\text { Descriptive } \\
\text { statistics } \\
\text { Paired t-test and } \\
\text { independent } \\
\text { t-test }\end{array}$ & $\begin{array}{l}\text { Factors significantly related to } \\
\text { adherence to sodium reduction }(\mathrm{p}< \\
0.05) \text { : Perceived benefits, perceived } \\
\text { barriers, perceived severity, perceived } \\
\text { susceptibility }\end{array}$ \\
\hline 2 & $\begin{array}{l}\text { Saleema, } \\
\text { Panpakdee, } \\
\text { Arpanantikul, \& } \\
\text { Chai-Aroon, } \\
\text { 2016. [14] }\end{array}$ & $\begin{array}{l}\text { A descriptive } \\
\text { cross-sectional } \\
\text { design }\end{array}$ & $\begin{array}{l}402 \text { Thais with } \\
\text { essential } \\
\text { hypertension } \\
\text { who received } \\
\text { treatment at } \\
\text { outpatient } \\
\text { medical clinics }\end{array}$ & $\begin{array}{l}\text { Knowledge about } \\
\text { hypertension, } \\
\text { Knowledge about self-care } \\
\text { demands, } \\
\text { Patient-provider } \\
\text { communication, } \\
\text { Duration of hypertension, } \\
\text { Family support, } \\
\text { Perception about } \\
\text { hypertension }\end{array}$ & $\begin{array}{l}\text { Self-report: The Self- } \\
\text { Care Behavior } \\
\text { Questionnaire } \\
\text { (SCBQ) which asked } \\
\text { about the frequency of } \\
\text { practicing self-care } \\
\text { activities for } \\
\text { hypertension control }\end{array}$ & $\begin{array}{l}\text { Structural } \\
\text { Equation } \\
\text { Modelling }\end{array}$ & $\begin{array}{l}\text { Factors significantly related to } \\
\text { adherence to self-care behaviors for } \\
\text { hypertension control }(\mathrm{p}<0.001) \text { : } \\
\text { Patient-provider communication, } \\
\text { knowledge about hypertension, } \\
\text { knowledge about self-care, and } \\
\text { perception about hypertension }\end{array}$ \\
\hline 3 & $\begin{array}{l}\text { Sangprasert, } \\
\text { Buranatrevedh, } \\
\text { \& Pipatsatitpong, } \\
\text { 2016. [15] }\end{array}$ & $\begin{array}{l}\text { Quasi- } \\
\text { experimental } \\
\text { study }\end{array}$ & $\begin{array}{l}36 \text { Thai adults } \\
\text { with essential } \\
\text { hypertension }\end{array}$ & Social support & $\begin{array}{l}\text { Self-report: } \\
\text { Preventive behavior } \\
\text { questionnaire and } \\
\text { diaries of home } \\
\text { behavior records }\end{array}$ & $\begin{array}{l}\text { Quantitative data: } \\
\text { Paired t-test } \\
\text { Qualitative data: } \\
\text { Content } \\
\text { describing from } \\
\text { interviews and } \\
\text { focus groups }\end{array}$ & $\begin{array}{l}\text { Factors significantly related to } \\
\text { adherence to preventive behaviors: } \\
\text { Social support }\end{array}$ \\
\hline 4 & $\begin{array}{l}\text { Leelacharas, } \\
\text { Chontichachalala } \\
\text { uk \& Snongdech, } \\
\text { 2015. [16] }\end{array}$ & $\begin{array}{l}\text { Descriptive and } \\
\text { cross-sectional } \\
\text { survey design }\end{array}$ & $\begin{array}{l}660 \text { Thais } \\
\text { diagnosed with } \\
\text { essential } \\
\text { hypertension } \\
\text { living in urban } \\
\text { and rural areas }\end{array}$ & $\begin{array}{l}\text { Sex, } \\
\text { Age, } \\
\text { Education level }\end{array}$ & $\begin{array}{l}\text { Self-report: } \\
\text { 1) Dietary Behavioral } \\
\text { Scale (DBS), } \\
\text { 2) Exercise } \\
\text { Behavioral Scale } \\
\text { (EBS) }\end{array}$ & $\begin{array}{l}\text { Descriptive } \\
\text { analysis, one-way } \\
\text { MANOVA, and } \\
\text { post hoc analysis }\end{array}$ & $\begin{array}{l}\text { Factors significantly related to } \\
\text { adherence to preventive behaviors ( } \mathrm{p} \\
<0.05 \text { ): Age and gender } \\
\text { Factors not significantly related to } \\
\text { adherence to preventive behaviors ( } \mathrm{p} \\
>0.05 \text { ): Educational level }\end{array}$ \\
\hline 5 & $\begin{array}{l}\text { Piaseu, } \\
\text { Thaothong, } \\
\text { Maruo, Jarupat, } \\
\text { \& Piyatrakul, } \\
\text { 2015. [17] }\end{array}$ & $\begin{array}{l}\text { Experimental } \\
\text { design }\end{array}$ & $\begin{array}{l}60 \text { Thais with } \\
\text { essential } \\
\text { hypertension in } \\
2 \text { communities } \\
\text { in central } \\
\text { Thailand }\end{array}$ & $\begin{array}{l}\text { Demographic } \\
\text { characteristics, } \\
\text { Community participation }\end{array}$ & $\begin{array}{l}\text { Self-report: Health } \\
\text { behavior } \\
\text { questionnaire for } \\
\text { persons with } \\
\text { hypertension }\end{array}$ & $\begin{array}{l}\text { Descriptivestatisti } \\
\text { cs,Chi-squaretest, } \\
\text { Fisher's } \\
\text { exactprobabilityte } \\
\text { st, t-test, Mann } \\
\text { Whitney U test, } \\
\text { and Wilcoxon } \\
\text { signed-rank test }\end{array}$ & $\begin{array}{l}\text { Factors significantly related to } \\
\text { adherence to stress management }(\mathrm{p}< \\
0.05) \text { : Community participation }\end{array}$ \\
\hline 6 & $\begin{array}{l}\text { Jianvitayakij, } \\
\text { Panpakdee, } \\
\text { Malathum, } \\
\text { Duffy, \& } \\
\text { Viwatwongkasea } \\
\text { m, 2014. [18] }\end{array}$ & $\begin{array}{l}\text { Cross-sectional, } \\
\text { predictive } \\
\text { design }\end{array}$ & $\begin{array}{l}266 \text { Thai men } \\
\text { with } \\
\text { hypertension } \\
\text { who smoked } \\
\text { cigarettes }\end{array}$ & $\begin{array}{l}\text { Age, } \\
\text { Education level } \\
\text { Nicotine dependence, } \\
\text { Concern about the risks of } \\
\text { smoking, } \\
\text { Perceived benefits, } \\
\text { Perceived barriers, } \\
\text { Perceived self-efficacy, } \\
\text { Social support }\end{array}$ & $\begin{array}{l}\text { Self-report: Smoking } \\
\text { cessation behavior }\end{array}$ & $\begin{array}{l}\text { Descriptive } \\
\text { statistics and } \\
\text { logistic } \\
\text { regression. }\end{array}$ & $\begin{array}{l}\text { Factors significantly related to } \\
\text { adherence to smoking cessation ( } \mathrm{p}< \\
0.05) \text { : Perceived self-efficacy and } \\
\text { concern about the dangers of } \\
\text { smoking, } \\
\text { Factors not significantly associated } \\
\text { with adherence to smoking cessation } \\
(\mathrm{p}>0.05) \text { : Age, education smoking } \\
\text { cessation, nicotine dependence, } \\
\text { perceived benefits, perceived barriers } \\
\text { and social support }\end{array}$ \\
\hline 7 & $\begin{array}{l}\text { Pinprapapan, } \\
\text { Panuthai, } \\
\text { Vannarit, \& } \\
\text { Srisuphan, } 2013 . \\
\text { [19] }\end{array}$ & $\begin{array}{l}\text { Cross-sectional } \\
\text { design }\end{array}$ & $\begin{array}{l}321 \text { Thais with } \\
\text { essential } \\
\text { hypertension }\end{array}$ & $\begin{array}{l}\text { Health beliefs, } \\
\text { Knowledge about } \\
\text { hypertension, } \\
\text { Social support, } \\
\text { Provider-patient } \\
\text { communication, } \\
\text { Perceived self-efficacy }\end{array}$ & $\begin{array}{l}\text { Self-report: The } \\
\text { Hypertensive } \\
\text { Adherence to } \\
\text { Therapeutic Regimens } \\
\text { Scale }\end{array}$ & $\begin{array}{l}\text { Pearsons' product } \\
\text { moment } \\
\text { correlation } \\
\text { coefficient and } \\
\text { structural } \\
\text { equation } \\
\text { modeling }\end{array}$ & $\begin{array}{l}\text { Factors significantly related to } \\
\text { adherence to therapeutic regimens ( } \mathrm{p} \\
<0.01 \text { ): Social support, provider- } \\
\text { patient communication, and perceived } \\
\text { self-efficacy and health belief, } \\
\text { Factors not significantly associated } \\
\text { with adherence to therapeutic } \\
\text { regimens }(\mathrm{p}>0.05) \text { : Knowledge } \\
\text { about hypertension }\end{array}$ \\
\hline
\end{tabular}




\begin{tabular}{|c|c|c|c|c|c|c|c|}
\hline $\mathbf{S} / \mathbf{N}$ & Author (year) & Study design & Sample & Independent variable & $\begin{array}{c}\text { Adherence to } \\
\text { preventive behaviors } \\
\text { measure }\end{array}$ & Data analysis & Results \\
\hline 8 & $\begin{array}{l}\text { Srison, } \\
\text { Masingboon, \& } \\
\text { Duangpaeng, } \\
\text { 2011. [20] }\end{array}$ & $\begin{array}{l}\text { Cross-sectional } \\
\text { design }\end{array}$ & $\begin{array}{l}130 \text { Thais with } \\
\text { essential } \\
\text { hypertension }\end{array}$ & $\begin{array}{l}\text { Demographic } \\
\text { characteristic, } \\
\text { Client-professional } \\
\text { interaction }\end{array}$ & $\begin{array}{l}\text { Self-report: } \\
\text { Adherence to } \\
\text { treatment } \\
\text { questionnaires }\end{array}$ & $\begin{array}{l}\text { Descriptive } \\
\text { statistics and } \\
\text { Pearson's product } \\
\text { moment } \\
\text { correlation } \\
\text { coefficient } \\
\end{array}$ & $\begin{array}{l}\text { Factors significantly related to } \\
\text { adherence to lifestyle modification ( } \mathrm{p} \\
<0.01 \text { ): Decision control, provision } \\
\text { of health information and professional } \\
\text { competency, }(0.05) \text { : Affective support }\end{array}$ \\
\hline 9 & $\begin{array}{l}\text { Houmkhuntod \& } \\
\text { Preechawong, } \\
\text { 2009. [21] }\end{array}$ & $\begin{array}{l}\text { Quasi- } \\
\text { experimental } \\
\text { research }\end{array}$ & $\begin{array}{l}60 \text { Thais with } \\
\text { essential } \\
\text { hypertension } \\
\text { receiving care } \\
\text { at a } \\
\text { hypertension } \\
\text { clinic of a } \\
\text { community } \\
\text { hospital } \\
\end{array}$ & Perceived self-efficacy & $\begin{array}{l}\text { Self-report: Dietary } \\
\text { consumption } \\
\text { and exercise behaviors } \\
\text { questionnaire }\end{array}$ & $\begin{array}{l}\text { Descriptive } \\
\text { statistics and } \\
\text { paired sample } \\
\text { t-test, and } \\
\text { independent t-test }\end{array}$ & $\begin{array}{l}\text { Factors significantly related to dietary } \\
\text { consumption and exercise behaviors } \\
(p<0.05) \text { : Perceived self-efficacy }\end{array}$ \\
\hline 10 & $\begin{array}{l}\text { Limcharoen, } \\
\text { Masingboom \& } \\
\text { Kunsongkeit, } \\
\text { 2007. [22] }\end{array}$ & $\begin{array}{l}\text { Descriptive } \\
\text { correlational } \\
\text { research }\end{array}$ & $\begin{array}{l}248 \text { Thais with } \\
\text { essential } \\
\text { hypertension }\end{array}$ & $\begin{array}{l}\text { Demographic } \\
\text { characteristic, } \\
\text { Knowledge about } \\
\text { hypertension, } \\
\text { Healthcare service }\end{array}$ & $\begin{array}{l}\text { Self-Report: } \\
\text { Adherence to } \\
\text { treatment } \\
\text { questionnaires }\end{array}$ & $\begin{array}{l}\text { Descriptive } \\
\text { statistics } \\
\text { Spearman's rank } \\
\text { correlation } \\
\text { coefficient }\end{array}$ & $\begin{array}{l}\text { Factors significantly related to } \\
\text { adherence to lifestyle modification ( } \mathrm{p} \\
<0.05) \text { : Age, knowledge of } \\
\text { hypertension }\end{array}$ \\
\hline 11 & $\begin{array}{l}\text { Tantayothin, } \\
\text { 2004. [23] }\end{array}$ & Cross-sectional & $\begin{array}{l}180 \text { Thais with } \\
\text { essential } \\
\text { hypertension }\end{array}$ & $\begin{array}{l}\text { Perceived self-efficacy, } \\
\text { Perceived benefits, } \\
\text { Perceived barriers, } \\
\text { Marital status }\end{array}$ & $\begin{array}{l}\text { Self-report: } \\
\text { Nutritional and } \\
\text { exercise behaviors } \\
\text { questionnaire }\end{array}$ & $\begin{array}{l}\text { Pearson's product } \\
\text { moment } \\
\text { correlation } \\
\text { coefficient, and } \\
\text { stepwise multiple } \\
\text { regression }\end{array}$ & $\begin{array}{l}\text { Factors significantly related to } \\
\text { adherence to dietary control }(\mathrm{p}< \\
0.001) \text { : Perceived self-efficacy, } \\
\text { perceived benefits, and marital status, } \\
\text { Factors significantly related to } \\
\text { adherence to exercise behaviors ( } \mathrm{p}< \\
0.001) \text { : Perceived self-efficacy, } \\
\text { perceived benefits, and perceived } \\
\text { barriers }\end{array}$ \\
\hline 12 & $\begin{array}{l}\text { Naewbood, } \\
\text { Sorajjakool \& } \\
\text { Triamchaisri, } \\
\text { 2012. [25] }\end{array}$ & $\begin{array}{l}\text { Qualitative } \\
\text { research }\end{array}$ & $\begin{array}{l}15 \text { Thais in } \\
\text { Southern } \\
\text { California } \\
\text { between the } \\
\text { ages of } 45-95 \\
\text { who had been } \\
\text { diagnosed with } \\
\text { hypertension }\end{array}$ & $\mathrm{N} / \mathrm{A}$ & None & & $\begin{array}{l}\text { Religion plays an important role by } \\
\text { helping hypertensive persons to } \\
\text { manage their stress level and offering } \\
\text { them beneficial instruction about } \\
\text { health practices such as diet and } \\
\text { exercise }\end{array}$ \\
\hline 13 & $\begin{array}{l}\text { Huangtong, } \\
\text { Piaseu, \& } \\
\text { Kaveevivitchai, } \\
\text { 2013. [26] }\end{array}$ & Case study & $\begin{array}{l}1 \text { man and } 1 \\
\text { woman }\end{array}$ & $\mathrm{N} / \mathrm{A}$ & None & Content analysis & $\begin{array}{l}\text { Themes identified } 4 \text { areas of } \\
\text { problems and barriers; } \\
\text { (1) individual and family, } \\
\text { (2) interaction within family and } \\
\text { society, } \\
\text { (3) community, } \\
\text { (4) community beliefs and cultural } \\
\text { practices }\end{array}$ \\
\hline 14 & $\begin{array}{l}\text { Kirdphon, } 2003 . \\
\text { [27] }\end{array}$ & $\begin{array}{l}\text { Grounded } \\
\text { theory study }\end{array}$ & $\begin{array}{l}19 \text { people with } \\
\text { essential } \\
\text { hypertension }\end{array}$ & N/A & None & $\begin{array}{l}\text { Constant } \\
\text { comparative } \\
\text { method }\end{array}$ & $\begin{array}{l}\text { Patients' perception of hypertension } \\
\text { influenced their management } \\
\text { behaviors for hypertension, } \\
\text { There was a communication problem } \\
\text { between providers and clients that } \\
\text { contributed to the clients' inability to } \\
\text { manage their condition appropriately, } \\
\text { Acceptance of and adjustment to the } \\
\text { chronicity of the disease } \\
\text { have been recognized as important } \\
\text { factors in living with and managing } \\
\text { hypertension }\end{array}$ \\
\hline
\end{tabular}




\section{Perceived benefits}

Patients who perceived the benefits of lifestyle changes were more likely to adhere to a healthy diet and physical activity in addition to engaging in abstinence from alcohol or smoking. A total of 4 quantitative studies have examined hypertensive Thais' beliefs about the perceived benefits of preventive behaviors and adherence $[13,18,19,23]$. Two of cross-sectional studies revealed that perceived benefits was a significant predictor of dietary control and exercise behaviors [23] as well as lifestyle modifications in essential hypertensive patients [19]. This is consistent with a study by Chonsin et al. [13] where they found that perceived benefits of sodium reduction to control hypertension was associated with adherence to preventive behavior recommendations. On the other hand, Jianvitayakij et al. [18] found that perceived benefits did not significantly predict smoking cessation. Although this study reported high levels of perceived benefits, and thus it might have made less contribution in smoking cessation. The probable reasons could be that this study used a non-probability sample in collecting data, so the generalizability may be limited [18]. These quantitative studies measured adherence to preventive behaviors using selfreport questionnaires $(\mathrm{n}=2)$, self-report of food intake during the previous $24 \mathrm{~h}(\mathrm{n}=1)$, and smoking cessation behavior $(\mathrm{n}=1)$.

\section{Perceived barriers}

Studies on perceived barriers $(n=5)$ reported that they are associated with adherence to preventive behaviors. Four quantitative studies revealed that perceptions about barriers to following prescribed preventive behaviors had a relationship to adherence regarding preventive regimens of hypertensive Thai adults $[13,18,19,23]$. One qualitative study supported the idea that 4 barriers consisting of individual and family, interaction within family and society, community, and community beliefs and cultural practices are the main factors influencing adherence to preventive behaviors [26]. Researchers assessed adherence to preventive behaviors with self-report measure $(n=3)$ using recorded smoking cessation behavior, food intake during the previous $24 \mathrm{~h}$, and questionnaires.

\section{Perceived self-efficacy}

Among the patient-related factors correlating to adherence to preventive behaviors, much attention is currently being focused on the role of the individuals' perceived self-efficacy, the individual's degree of confidence in his or her ability to engage in a healthy behavior change. In a cross-sectional study, 3 studies found a positive correlation between preventive behaviors adherence rates and perceived selfefficacy of hypertensive Thai adults $[18,19,23]$. The finding were supported by a quasi- experimental study, who found that perceived self-efficacy were significantly associated with adherence to dietary recommendation and exercise behaviors [21]. Most quantitative studies which examined perceived selfefficacy factors assessed adherence using self-reported questionnaires $(n=4)$.

\section{Thai cultural values}

Cultural values play an important role in determining adherence to preventive behaviors. Culture values as a conception of "the desirable" [24]. Three qualitative studies found that culture influences patients' health behaviors [25-27]. Huangtong and colleagues [26] discovered that attitudes towards folkways such as local dietary habits and a tradition of alcohol consumption were problems for Thais when participating in lifestyle modifications. Moreover, Kirdphon (2003) [27] reported that Thai patients who were able to accept that they have hypertension used flexibility and adjustment to integrate preventive behavior recommendations into their daily lives because they believe that health is base on a balance of illness control activities and social activities. They believe that this balance is important for maintaining health. Furthermore, Naewbood and colleagues [25] revealed that religion plays an vital role in helping hypertensive Thai adults adhere to stress management.

\section{Health team and health system related factors}

Health team and health system related factors are significant predictors of adherence to preventive behaviors, especially patient-provider relationships. Five studies identified and/or examined patientsprovider communication $[14,17,19,20,27]$. The interpersonal dynamics of the patient-provider 
http://wjst.wu.ac.th

relationship play a key role in helping patients to adhere to preventive behaviors of hypertensive Thai adults. Five studies found that patient-provider communication and relationships were associated with adherence to preventive behavior recommendations which resulted in increased patient satisfaction and decreased patient stress. Most of the research related to patient-provider communication factors has been quantitative, and has reported that a perception of good communication with healthcare providers had a positive effect on adherence to preventive behaviors [14,17,19,20]. The findings from one qualitative study showed that communication problems contribute to the patients' inability to manage their condition appropriately [27]. Studies examining adherence to preventive behaviors using self-reported questionnaires $(n=4)$.

\section{Knowledge gaps and methodological limitations in the literature}

There are knowledge gaps and methodological limitations across the research studies. Firstly, this study discovered that more research is needed to better understand which factors are associated with adherence to preventive behaviors of Thai adults with hypertension. The primary factor of concern in these studies, as supported by the qualitative designs, was Thai cultural values. This finding should be interpreted with caution due to the limited number of studies available and because the qualitative study did not recruit a large enough number of informants necessary to provide rich information. Further research is needed to confirm whether these associations exist.

Secondly, other factors, such as duration of hypertension and perceived susceptibility, have been widely recognized as important to consider when providing preventive treatment to Thais with hypertension $[13,14]$. However, the number of studies conducted exploring factors relating to adherence regarding preventive behaviors in hypertensive Thais is insufficient.

Thirdly, the lack of suitable adherence to preventive behavior measures, the lack of long-term and carefully monitored outcome studies, and limited attention to related research designs make it difficult to support or refute any of these findings. Additionally, many studies measured adherence to preventive behaviors using self-report questionnaires. Without standardized definitions of the concept of adherence, the majority of researchers measure the elements of this concept differently. Substantial heterogeneity may reflect the differences in methods of defining adherence to preventive behaviors across the studies.

Fourthly, the major drawback of this study is that most of the previous researches were crosssectional designs. There is a need for more longitudinal designs in order to study adherence to preventive behaviors over more than one point in time because adherence to preventive behaviors is a multidimensional concept and is not a static state but rather, is dynamic. It cannot be determined that adherence to preventive behaviors and factors affecting adherence to preventive behaviors are stable where adopting new lifestyle habits does not automatically lead to sustained the changes.

Lastly, much of the literature specifically addresses factors associated with preventive behaviors. Additionally, those related studies that do exist are predominantly short-term studies with no reliable measure of adherence. Despite these limitations, the factors identified are useful for community health care nurses in designing interventions to increase adherence to preventive behaviors.

\section{Discussion}

This study intended to analyze empirical findings that identified factors influencing adherence to lifestyle modifications of Thai adults with hypertension. The findings found 8 factors that consistently affected adherence to preventive behaviors. These factors include social support, knowledge of hypertension, perception of illness, perceived benefits, perceived self-efficacy, patient-provider communication, and Thai cultural values and can be manipulated by the healthcare provider for development of a program to enhance adherence behaviors.

Social support is consistently well documented as having a favorable effect on adherence to lifestyle modification practices. A previous meta-analysis by Magrin et al. [28] has highlighted that functional social support is significantly and positively related to adherence to healthy lifestyle in hypertensive patients. Functional social support, such as practical support, emotional support, and information-based 
support, is potentially important in helping promote patients' willingness to initiate and maintain lifestyle change recommendations. This is because lack of adequate information and occasional stress, are common reasons for non-adherence. Additionally, it was found that lack of adequate support from spouse and/or family was associated with lower rates of adherence to dietary recommendations [29]. Moreover, peer groups verified to be one of the effective ways to offer support and sense of hope to the patients. Therefore, due to these reasons hypertensive Thai patients with greater social support tend to be more adherent to preventive behaviors.

Hypertensive patients who had adequate knowledge of hypertension were more adherent to lifestyle change than those who did not [30]. This is likely because knowledge of hypertension helps patients understand that the disease needs long term treatment. Poor adherence to preventive behaviors might be due to its asymptomatic symptoms.

Perception of disease among Thais with hypertension is consistent with western literature in which hypertension has been described as an asymptomatic disease characterized. Patients still consider themselves as healthy when no signs and symptoms of hypertension have been observed [31]. Previous studies have confirmed that adherence is significantly and positively correlated with the patients' beliefs in the severity of the disease to be prevented or treated [32,33]. Studies revealed that negative perceptions were associated with non-adherence to lifestyle modifications [34]. Therefore, assessing the patient's views of the asymptomatic nature of hypertension may help their adaptive process.

Perceived benefits and perceived barriers were found to be associated with adherence to preventive behaviors. Hypertensive patients do not engage in preventive behavior recommendations due to their perception of there being few benefits but many barriers to preventive behaviors. In fact, patient's behavior is determined by evaluation of perceived positive and negative forces on their health behavior. According to the health belief model, the presence of both perceived barriers and benefits of preventive behaviors leads to the likelihood of engaging in preventive health practices [35].

Perceived self-efficacy is a major determinant of adherence to preventive behaviors. This can be explained through Social Cognitive Theory [36,37]. This theory posits that if an individual's perceived capability to engage in preventive activities regularly is high, they are more likely to perform preventive activities [36]. Additionally, increasing perceived self-efficacy has been show to play a key role in predicting adherence to preventive behaviors during difficult events such as making an effort to incorporate preventive behaviors into daily life [38-40].

This review has demonstrated and confirmed that adherence to preventive behaviors is influenced by community beliefs and cultural values. Since participation in adherence to preventive behaviors is part of everyday life with the disease, Thais will follow preventive behavior recommendations strictly when they believe that disease impacts on their lives or has power over them [41]. However, non-adherence to preventive behaviors might be due to Thais having low motivation to work hard to achieve their ideal goals [42]. This means that there are numerous ways that an individual's resources and environment may be inadequate for carrying out the preventive activities. Additionally, Thais make decisions regarding adherence to preventive behaviors based on their personal comfort [27]. Several studies found that when Thai patients perceive hypertension as a permanent illness that they have to live with, flexibility and adjustment are used to enable them to adhere to tasks to control their hypertension $[41,43]$. Therefore, the Thai cultural values of holism and gradual adjustment are consistently found to be used to construct adherence tasks to help patients control their disease [44].

Patient-provider communication is a further factor that plays an important role in determining adherence lifestyle modification outcomes. Research evidence indicates that effective patient-provider communication promotes the development of a satisfactory relationship, encourages patient and physician to engage in appropriate information exchange, and tends to be more motivated to adhere $[45,46]$. A perception of good communication with physicians encourages patients to be involved in their own care and therefore they tend to be more motivated to adhere [45]. Poor communication between patients and healthcare providers is associated with poorer adherence to preventive behaviors in patients with hypertension [47]. Cohesive partnerships and effective interpersonal communication should provide benefits to patients following mutually agreed-upon recommendations. 
http://wjst.wu.ac.th

\section{Implications of this review}

Findings from this review can be useful for community health nurses in designing interventions based on these factors reported within. An increased understanding will help nurses in modifying their intervention to fit their patients' lifestyles and improve adherence to preventive behaviors among Thai adults with hypertension. Perception of disease is useful to understand what hypertension means to Thais in terms of their everyday life. In order to develop an effective community health education program to encourage adherence to preventive behaviors, the program has to motivate Thai adults to be aware of the risk factors for complications associated with hypertension and help them in developing an appropriate understanding of hypertension and treatment plan.

Perceived self-efficacy is closely linked to adherence to preventive behaviors. Community health nurses can provide interventions for their clients regarding their preventive behavior treatment by increasing the individual's confidence in his or her ability to initiate and maintain preventive behaviors. Helping patients to understand that adherence to preventive behavior treatment is manageable can increase their confidence in their ability to live with hypertension.

Social support is associated with adherence regarding preventive behaviors. Programs involved can also be emphasized by involving family members in participating in preventive behavior practices because social support has potential benefits in helping patients positively initiate and sustain any behavioral change.

Based on this review, it is clear that a perception of good communication with physicians encourages Thais with hypertension to be followed in their preventive behavior advices and therefore to be more motivated to adhere. Since good communication in health care settings is highly correlated with better patient adherence, promoting effective communication in a healthcare environment is essential and challenging. Nurse and other health care providers should receive regular communication skills training in order to improve patient care and relationships. Additionally, the willingness of the nurse to form a 'doctor-patient team' is important.

A number of Thai cultural values and practices are related to adherence to preventive behaviors. Since adherence to preventive behaviors is identified in ways consistent with the Thai cultural context that governs everyday life; strategies involving the notions of holism, autonomy and gradual adjustment may provide practical guidelines for adherence to preventive behaviors of Thais with hypertension.

\section{Strengths and limitations}

The strengths of this review are that it was composed of both quantitative and qualitative studies along with an extensive search through various databases in order to gain an in-depth information on the phenomenon of interest. Tables of the findings were presented in this review.

However, this review has its limitations. First, this literature does not identified all unpublished studies that may alter the true effect of factors associated with adherence to preventive behaviors due to publication bias. Second, there was a lack of information on facilitators for adherence regarding moderation of alcohol intake.

\section{Conclusions}

Overall, this review sought to examine empirical findings that identified factors which affected adherence to preventive behaviors. These factors are essential for developing interventions to increase the level of adherence to preventive behaviors of Thai adults. Recommendations for future interventions and research for Thais with hypertension were also presented.

\section{Acknowledgements}

The author wishes to thank Boromarajonani College of Nursing, Songkhla, for its support. 
http://wjst.wu.ac.th

\section{References}

[1] P Muntner, BR Davis, WC Cushman, S Bangalore, DA Calhoun, SL Pressel, HR black, JB Kostis, JL Probstfield, PK Whelton and M Rahman. Treatment-resistant hypertension and the incidence of cardiovascular disease and end-stage renal disease: Results from the antihypertensive and lipidlowering treatment to prevent heart attack trial (ALLHAT). Hypertension 2014; 64, 1012-21.

[2] Y Wang, J Xu, X Zhao, D Wang, C Wang, L Liu, A Wang, X Meng, H Li and Y Wang. Association of hypertension with stroke recurrence depends on ischaemic stroke subtype. Stroke 2013; 44, 1232 7.

[3] NCD Risk Factor Collaboration. Worldwide trends in blood pressure from 1975 to 2015: A pooled analysis of 1479 population based measurement studies with 19.1 million participants. Lancet 2017, 389, 37-55.

[4] W Aekplakorn, R Sangthong, P Kessomboon, P Putwatana, R Inthawong, S Taneepanichskul, P Piyamitr, S Sangwatanaroj and S Chariyalertsak. Changes in prevalence, awareness, treatment and control of hypertension in Thai population, 2004-2009: Thai National Health Examination Survey III-IV. J. Hypertens. 2012; 30, 1734-42.

[5] W Aekplakorn. Thailand National Health Examination Survey V. Health System Research Institute, Nonthaburi, Thailand, 2016.

[6] AV Chobanian, GL Bakris, HR Black, WC Cushman, LA Green, JL Izzo, DW Jones, BJ Materson, S Oparil, JT Wright, JR Toccella and EJ Rocella. Seventh report of the Joint National Committee on prevention, detection, evaluation, and treatment of high blood pressure. Hypertension 2003; 42, 1206-52.

[7] S Vooradi and UV Mateti. A systemic review on lifestyle interventions to reduce blood pressure. $J$. Health Res. Rev. 2016; 3, 1-5.

[8] HO Dickinson, JM Mason, DJ Nicolson, F Campbell, FR Beyer, JV Cook, B Williams and GA Ford. Lifestyle interventions to reduce raised blood pressure: A systematic review of randomized controlled trials. J. Hypertens. 2006; 24, 215-33.

[9] E Sabate. Adherence to Long-Term Therapies: Evidence for Action. Geneva, Switzerland, World Health Organization, 2003.

[10] S Chongthawonsatid. Demographic factors and health care behavior of hypertension disease in Thailand (in Thai). Silpakorn Univ. Sci. Tech. J. 2015; 9, 9-16.

[11] A Tibebu, D Mengistu and L Negesa. Adherence to recommended lifestyle modifications and factors associated for hypertensive patients attending chronic follow-up units of selected public hospitals in Addis Ababa, Ethiopia. Patient Prefer. Adher. 2017; 11, 323-30.

[12] Joanna Briggs Institute. Available at: http://joannabriggs.org/assets/docs/sumari/ReviewersManual Methodology-for-JBI-Scoping-eviews 2015 v2.pdf, accessed February 2017.

[13] P Chonsin, C Kaveevivitchai, N Neelapaichit and N Piaseu. Impact of a blood pressure regulating programme on health beliefs, health behaviour, amount of sodium intake and hypertension levels in community members with hypertension (in Thai). Thai J. Nurs. Council 2016; 31, 63-75.

[14] L Saleema, O Panpakdee, M Arpanantikul and T Chai-Aroon. The influence of basic conditioning factors and self-care agency on self-care behaviors in Thais with hypertension. Pac. Rim. Int. J. Nurs. Res. 2016; 20, 5-17.

[15] P Sangprasert, S Buranatrevedh and D Pipatsatitpong. The effectiveness of hypertensive management programs and social support in primary health care systems: Preliminary study. Siriraj Med. J. 2016; 68, 369-76.

[16] S Leelacharas, J Chontichachalalauk and W Snongdech. Dietary-taking, exercise, and medication adherence in Thais with hypertension based on gender, age, and educational level (in Thai). J. Public Health Nurs. 2015; 29, 56-70.

[17] N Piaseu, N Thaothong, S Jarupat Maruo and S Piyatrakul. Effects of community participation program for blood pressure control on health behaviors and blood pressure in community dwellers with hypertension. J. Hypertens. 2015; 33, e149. 
http://wjst.wu.ac.th

[18] S Jianvitayakij, O Panpakdee and P Malathum, S Duffy and C Viwatwongkasem. Factors influencing smoking cessation behavior among Thai male smokers with hypertension. Pac. Rim. Int. J. Nurs. Res. 2014; 18, 100-10.

[19] E Pinprapapan, S Panuthai, T Vannarit and W Srisuphan. Casual model of adherence to therapeutic regimens among Thais with hypertension. Thai J. Nurs. Res. 2013; 17, 268-81.

[20] P Srison, K Masingboon and S Duangpaeng. Relationship between client-professional interaction and adherence to treatment among persons with hypertension (in Thai). J. Facul. Nurs. Burapha Univ. 2011; 17, 60-74.

[21] T Houmkhuntod and S Preechawong. Effects of perceived self-efficacy promoting program on dietary consumption and exercise behaviors among hypertensive patients (in Thai). J. Nurs. Sci. Health 2009; 32, 19-28.

[22] S Limcharoen, K Masingboon and W Kunsongkeit. Factors related to adherence to treatment among essential hypertensive patients (in Thai). J. Facul. Nurs. Burapha Univ. 2007; 15, 63-79.

[23] S Tantayothin. 2004, Factors Influencing Nutritional and Exercise Behaviors of Hypertension Patients in Sainoi District. Master Degree, Dissertation. Mahidol University, Bankok, Thailand.

[24] VA Braithwaite and WA Scott. "Values," in Measures of Personality and Social Psychological Attitudes. In: JP Robinson, PR Shaver and LS Wrightsman (Eds.). Academic Press, Burlington, 1991, p. 661-776.

[25] S Naewbood, S Sorajjakool and SK Triamchaisri. The role of religion in relation to blood pressure control among a Southern California Thai population with hypertension. J. Relig. Health 2012; 51, 187-97.

[26] S Huangtong, N Piaseu and C Kaveevivitchai. Case study: Behaviors, problems, and barriers of blood pressure control in persons with severe hypertension in a Community. Ramathibodi Nurs. $J$. 2013; 19, 129-42.

[27] W Kirdphon. 2003, Accepting and Adjusting to Chronicity of Hypertension: A Grounded Theory Study in Thai People. Ph.D. Dissertation, University of Washington, United States.

[28] ME Magrin, M D Addario, A Greco, M Miglioretti, M Sarini, M Scrignaro, P Steca, L Vecchio and E Crocetti. Social support and adherence to treatment in hypertensive patients: A meta-analysis. Ann. Behav. Med. 2015; 49, 307-18.

[29] OS Ojo, SO Malomo, PT Sogunle and AM Ige. Blood pressure (BP) control and perceived family support in patients with essential hypertension seen at a primary care clinic in Western Nigeria. $J$. Fam. Med. Prim. Care 2016; 5, 569-75.

[30] M Kilic, T Uzunçakmak and H Ede. The effect of knowledge about hypertension on the control of high blood pressure. Int. J. Cardiovasc. Acad. 2016; 2, 27-32.

[31] ARA Rahman, JG Wang, GMY Kwong, DD Morales, P Sritara and R Sukmawan. Perception of hypertension management by patients and doctors in Asia: Potential to improve blood pressure control. Asia Pac. Fam. Med. 2015; 14, 2.

[32] MR DiMatteo, KB Haskard and SL Williams. Health beliefs, disease severity, and patient adherence: A meta-analysis. Med. Care 2007; 45, 521-8.

[33] K Park, S Cho and JK Bower. Changes in adherence to non-pharmacological guidelines for hypertension. PLoS One 2016; 11, e0161712.

[34] SO Ike, PN Aniebue and UU Aniebue. Knowledge and perceptions related to hypertension, lifestyle behavior modifications and challenges that facing hypertensive patients. IOSR J. Nurs. Health Sci. $2015 ; 4,15-26$.

[35] CJ Carpenter. A meta-analysis of the effectiveness of the health belief model variables in predicting behavior. Health Comm. 2010; 25, 661-9.

[36] A Bandura. Self-Efficacy: The Exercise of Control. Freeman, New York, 1997, p. 36-38.

[37] J Warren-Findlow, RB Seymour and LR Brunner Huber. The association between self-efficacy and hypertension self-care activities among African American adults. J. Comm. Health 2012; 37, 15-24.

[38] LL Lee, A Arthur and M Avis. Using self-efficacy theory to develop interventions that help older people overcome psychological barriers to physical activity: A discussion paper. Int. J. Nurs. Stud. 2008; 45, 1690-9. 
http://wjst.wu.ac.th

[39] S Byrne, D Barry and NM Petry. Predictors of weight loss success: Exercise vs dietary self-efficacy and treatment attendance. Appetite 2012; 58, 695-8.

[40] E Martinez, KL Tatum, M Glass, A Bernath, D Ferris, P Reynolds and R Schnoll. Correlates of smoking cessation self-efficacy in a community sample of smokers. Addict. Behav. 2010; 35, 175-8.

[41] O Panpakdee, W Sritanyarat and S Tanomsup. Self-care process in Thai people with hypertension: An emerging model. Thai. J. Nurs. Rese. 2003; 7, 121-36.

[42] S Naewbood, S Sorajjakool and SK Triamchaisri. The role of religion in relation to blood pressure control among a Southern California Thai population with hypertension. J. Relig. Health 2012; 51, 187-97.

[43] B Naemiratch and L Manderson. Control and adherence: Living with diabetes in Bangkok, Thailand. Soc. Sci. Med. 2006; 63, 1147-57.

[44] S Chukumnird, U Boonyasopun and P Jittanoon. Perspectives regarding adherence to preventive behaviors: A qualitative study of Thais with prehypertension. Pac. Rim. Int. J. Nurs. Res. 2016; 20, 106-18.

[45] KB Zolnierek and MR DiMatteo. Physician communication and patient adherence to treatment: A meta-analysis. Med. Care 2009; 47, 826-34.

[46] C Stavropoulou. Non-adherence to medication and doctor-patient relationship: Evidence from a European survey. Patient Educ. Couns. 2011; 83, 7-13.

[47] M Baumann, A Tchicaya, N Lorentz and EL Bihan. Impact of patients' communication with the medical practitioners, on their adherence declared to preventive behaviours, five years after a coronary angiography, in Luxembourg. PLoS One 2016; 11, e0157321. 